\title{
Proyecciones de clima regional sobre España: atmósfera. Evaluación del clima presente
}

\section{Juan Pedro Montávez ${ }^{1}$, Jesús Fernández ${ }^{2}$, Ana Casanueva ${ }^{2 *}$, José Manuel Gutiérrez ${ }^{3}$, Enrique Sánchez ${ }^{4}$}

\author{
${ }^{1}$ Grupo de Modelización Atmosférica Regional, Departamento de Física, Universidad de Murcia, España \\ ${ }^{2}$ Grupo de Meteorología, Dpto. Matemática Apl. y Ciencias de la Computación, Universidad de Cantabria, España \\ ${ }^{3}$ Grupo de Meteorología, Instituto de Física de Cantabria (CSIC-UC), España \\ ${ }^{4}$ Facultad de Ciencias Ambientales y Bioquímica, Universidad de Castila-La Mancha (UCLM), España \\ (*) Actualmente en la Oficina Federal de Meteorología y Climatología (MeteoSwiss), Suiza
}

\begin{abstract}
Introducción
Las proyecciones futuras de cambio climático se basan principalmente en simulaciones con modelos de circulación global (MCG) forzados por futuros escenarios de concentración de gases de efecto invernadero. Su relativamente vasta resolución horizontal hace que la representación de algunos procesos atmosféricos relevantes para el clima regional no sea realista. Por otro lado, los datos proporcionados no tienen la resolución espacial que a menudo se necesita para estudios de impacto y adaptación. Para superar estos problemas, se siguen dos enfoques principales de reducción de escala (downscaling) o regionalización: 1) la regionalización dinámica, basada en modelos climáticos regionales (MCR) alimentados por salidas de los MCG, y 2) la regionalización estadística (MRE) basada en establecer relaciones estadísticas entre la circulación a gran escala de MCG y el clima regional/local observado. En este contexto, la península ibérica es un candidato perfecto para los ejercicios de regionalización, debido a la complejidad de su orografía y a su posición respecto a la trayectoria de los ciclones extratropicales, lo que produce una alta heterogeneidad espacial de las condiciones climáticas.
\end{abstract}

El primer paso al aplicar una técnica de regionalización es verificar su capacidad para reproducir el clima observado. Este es un paso fundamental, ya que nos informa sobre la confianza que podemos tener cuando el método/modelo se aplica a escenarios de cambio climático (Sánchez et al., 2011, entre muchos otros), clima pasado (Gómez-Navarro et al., 2011), o la generación de una base de datos pseudoreales (hindcast regional) para multitud de aplicaciones (Sotillo et al., 2005; Jerez et al., 2013c). Por otro lado, la evaluación del valor añadido con respecto al modelo global también es importante, porque la regionalización en los estudios climáticos solo tiene sentido si se proporciona información adicional y valiosa (Sánchez et al., 2011; Jiménez-Guerrero et al., 2013; Lorente-Plazas et al., 2015).
La evaluación climática actual de las técnicas de regionalización se puede realizar bajo dos perspectivas diferentes, dependiendo de los datos que guíen al modelo regional. Por un lado, el uso de datos cuasi-observacionales (reanálisis o análisis) permite evaluar la destreza del MCR directamente. Por otro lado, la utilización de MCG (proyecciones históricas) en las condiciones actuales permite evaluar el rendimiento de acoplamientos MCG-MCR particulares. Esto es crucial para las distintas aplicaciones en estudios de cambio climático, ya que cualquier deficiencia en la representación del presente puede afectar la credibilidad de los cambios futuros que se proyecten (Turco et al., 2013). La evaluación generalmente se realiza comparando distintos estadísticos climáticos (media, desviación estándar, así como otros índices) de los datos simulados y observados de referencia. Para simulaciones conducidas por reanálisis, la correspondencia diaria con observaciones se ha utilizado en algunos casos para evaluar los aspectos temporales de las simulaciones (para más detalles véase Maraun et al., 2015).

En este artículo se presenta un resumen del progreso reciente en la evaluación de métodos de regionalización dinámicos y estadísticos en España. El objetivo es actualizar los resultados de la evaluación previa de CLIVAR-España (Sánchez y Miguez-Macho, 2010), que se basó principalmente en los resultados del proyecto europeo PRUDENCE. Los estudios mostrados en este trabajo se basaron en los resultados de posteriores iniciativas internacionales de regionalización como ENSEMBLES (Déqué et al., 2012), VALUE (Maraun et al., 2015) y CORDEX (Jacob et al., 2014), así como de acciones estratégicas españolas para la generación coordinada de proyecciones regionales utilizando tanto métodos dinámicos (proyecto ESCENA) como estadísticos (proyecto ESTCENA). Estos proyectos han proporcionado simulaciones climáticas regionales con una resolución que varía de $0,44^{\circ}$ a $0,11^{\circ}$ (siendo la resolución típica de los MCG alrededor de $2^{\circ}$ ). 


\section{Regionalización dinámica}

Desde 2010 se han publicado multitud de estudios analizando los MCR en las condiciones climáticas actuales en la península ibérica. Algunos ejemplos son iniciativas y proyectos internacionales como EURO-CORDEX y Med-CORDEX o ENSEMBLES o el proyecto nacional ESCENA. Estos se pueden dividir en dos grupos: aquellos que se centran en la península ibérica o España (Herrera et al., 2010; Jiménez-Guerrero et al., 2013; Jerez et al, 2013b, entre otros), y aquellos en los que la península ibérica es solo una parte del área analizada (Vautard et al., 2013; Kotlarski et al., 2014; Katragkou et al., 2015, entre otros).

La mayoría de los estudios que evalúan los MCR generalmente se han centrado en la temperatura cerca de la superficie y la precipitación (Herrera et al., 2010; Jerez et al., 2013b; Gómez-Navarro et al., 2013), otros se han centrado en el viento (Jiménez et al., 2010; Lorente-Plazas et al., 2015; Gómez et al., 2016), la nieve (Pons et al., 2016), la radiación (Ruiz-Arias et al., 2013), o incluso los flujos entre la atmósfera y la superficie (Knist et al., 2017). Cabe mencionar que la evaluación de varias variables involucradas en un proceso es altamente recomendable pues permite identificar y evitar posibles compensaciones de errores (García-Díez et al., 2015b). Es importante prestar atención a los procesos en lugar o además de las variables. Por ejemplo, si se está interesado en la precipitación o el cambio de precipitación, hay que analizar el proceso (¿cómo/cuándo llueve?, ¿la cubierta de nubes es realista?, la separación de precipitación estratiforme y convectiva, etc.) para así poder contrastar nuestra confianza en el modelo. El potencial de la evaluación de procesos es una de las principales ventajas de los MCR respecto a los MRE. Otra dimensión a evaluar en los modelos es el régimen climático: evaluar la capacidad de reproducir el clima promedio (ciclo anual y variabilidad temporal) (Fernández et al., 2007; Argüeso et al., 2011; Jiménez-Guerrero et al., 2013; López-Franca et al., 2013) junto con regímenes de extremos (Argüeso et al., 2012; Domínguez et al., 2013; Vautard et al., 2013; López-Franca et al., 2015).

La principal conclusión de dichos estudios es que los MCR, en el caso particular de la península ibérica, proporcionan información muy valiosa, mejorando la distribución espacial regional y local de variables climáticas, debido principalmente a una mejor representación de las características de la superficie y la orografía. Los MCR son ampliamente capaces de capturar los regímenes espacio-temporales de precipitación, temperatura y viento, así como eventos extremos. Sin embargo, se observan sesgos sustanciales que dificultan la aplicabilidad directa de los resultados de los ejercicios con MCR en distintos sectores (hidrología, agricultura, energía, etc.). Esto abre un tema controvertido acerca de la corrección o ajuste del sesgo de los MCR, y su impacto sobre la señal del cambio climático (Casanueva et al., 2018; Turco et al., 2017). Además, los beneficios de aumentar la resolución en los MCR no son evidentes (por ejemplo, de $0,44^{\circ}$ a $0,11^{\circ}$ ). Mientras algunos estudios afirman que hay beneficios claros (Prein et al., 2016), otros (García-Díez et al., 2015a) sugieren que cuando la resolución aumenta, los índices de bondad tradicionales pueden no ser apropiados, o incluso indican que no hay una clara mejora, como por ejemplo en los sesgos medios del modelo (Casanueva et al., 2016b). Algunos resultados apuntan a que la formulación del modelo podría ser más importante que la resolución a la hora de tener una simulación más adecuada de ciertos procesos (Gaertner et al., 2016).

Los datos observacionales (y la falta de ellos) juegan un papel clave en la evaluación de los modelos. Podemos encontrar dos fuentes principales de datos observacionales: datos meteorológicos en bruto (estaciones, sondeos, datos satelitales, etc.) y datos en rejilla basados en los anteriores. Los segundos son más comúnmente utilizados para la evaluación de MCR. Hay varias bases de datos en rejilla disponibles para España, como E-OBS, CRU y Spain02 (Herrera et al., 2016). Estas se han construido usando distintos conjuntos de estaciones meteorológicas, así como diferentes métodos de interpolación. Esto genera diferencias importantes entre ellas, lo que hace que la evaluación del modelo sea dependiente de la propia base de datos que se escoja (Gómez-Navarro et al., 2012). Por lo tanto, las bases de datos observacionales son una fuente importante de incertidumbre en la evaluación del modelo. Se han empleado varias metodologías para comparar modelos y datos observacionales: mediante comparación directa y usando regionalización (agrupamiento) de datos. La primera consiste en comparar el valor observado con los datos del modelo interpolados en dicha ubicación (Fernández et al., 2007; Jiménez-Guerrero et al., 2013). Aquí surge cierta controversia pues los resultados pueden depender del método de interpolación seleccionado o incluso estar afectados por parámetros muy locales. La segunda se basa en la selección de regiones espaciales con variabilidad temporal similar para la comparación (Herrera et al., 2010; Argüeso et al., 2011; Lorente-Plazas et al., 2015; Argüeso et al., 2011). Esta técnica parece ser más robusta ya que los efectos locales que los MCR no pueden explicar se filtran pero, por otro lado, la pérdida de detalles regionales podría ocultar el valor añadido de los experimentos de alta resolución.

La regionalización dinámica lleva inherentemente una serie de incertidumbres relacionadas con prácticamente cualquier cambio en el modelo: núcleo dinámico, parametrizaciones físicas, tamaño y posición del dominio, así como de las condiciones iniciales. Estas incertidumbres generalmente se exploran utilizando conjuntos de simulaciones. Varios estudios han analizado la incertidumbre introducida por muchos de estos factores, siendo los conjuntos multimodelo más comunes (Herrera et al., 2010; Jiménez-Guerrero et al., 2013; Domínguez et al., 2013; Vautard et al., 2013). Para la península ibérica también se han llevado a cabo varios estudios sobre conjuntos multifísica (Fernández et al., 2007; Argüeso et al., 2011; Argüeso et al., 2012; García-Díez et al., 2013; Jerez et al., 2013b; Katragkou et al., 2015). Los conjuntos multi-física tienen la ventaja de perturbar un solo componente del modelo (microfísica, convección, radiación, etc.), vinculando así las diferencias a procesos específicos. De esta forma, se puede evaluar la contribución de los diferentes componentes a la incertidumbre total, y también los posibles cambios en su rol en el clima futuro (Jerez y col., 2013a). 
En la mayoría de los casos, las propiedades medias del conjunto muestran una gran mejora en la variabilidad temporal y la representación de los patrones espaciales de las variables climáticas frente a los miembros individuales, y muy a menudo la dispersión del conjunto suele cubrir los datos observados. Los conjuntos multifísica muestran una dispersión en los resultados comparable a la obtenida en conjuntos multimodelo, lo que sugiere que la distribución de conjuntos multimodelo se puede atribuir en gran medida a la diferente física de los modelos individuales. Otro resultado recurrente es que ningún miembro del conjunto, tanto en experimentos multifísica como en multimodelo, supera a los demás en todas las situaciones (tiempo y espacio) y los sesgos medios son independientes de la habilidad de reproducir la variabilidad temporal. Sin embargo, algunos esquemas o modelos particulares muestran un mejor rendimiento general, mientras que otros presentan peores resultados. Además, al evaluar modelos o parametrizaciones físicas analizando periodos largos (climatológicos) se concluye que los estudios a corto plazo deben usarse con precaución. Esto abre la puerta al problema de la destilación, es decir, poder decidir cómo las propiedades de los conjuntos deben ponderarse partiendo de mensajes contradictorios de diferentes modelos, configuraciones o experimentos para proporcionar información procesable. En los últimos años, la comunidad de MCR está incorporando más complejidad a sus modelos (principalmente componentes atmosféricos) acoplándolos a modelos de otros componentes del sistema climático a escala regional. Algunos ejemplos son los estudios de sensibilidad sobre el papel de los modelos de superficie terrestre (Jerez et al., 2010; Jerez et al., 2012; Knist et al., 2017) o el acoplamiento a modelos oceánicos regionales (Gaertner et al., 2016) y modelos de química atmosférica (Palacios-Peña et al., 2017). Si bien se demuestra que el papel del modelo de tierra es crucial para una simulación climática correcta, especialmente en áreas con un régimen de transición en la retroalimentación tierraatmósfera, no se encuentran trabajos que presenten claramente un impacto notable de acoplamiento de MCR a modelos oceánicos regionales. La mejora de las simulaciones climáticas por el acoplamiento de MCR a modelos de química no se ha experimentado completamente, aunque hay algunos resultados prometedores (Baró et al., 2017).

\section{Regionalización estadística}

Se pueden seguir dos enfoques fundamentales en el marco de la regionalización estadística. Bajo el enfoque Perfect Prog (PP), las relaciones estadísticas se calibran considerando observaciones tanto para los predictandos (observaciones históricas) como para los predictores (datos de reanálisis), mientras que las predicciones del modelo (MCG o MCR) se usan como predictores bajo la estrategia de estadísticas de salidas del modelo (MOS). El predictor típico en MOS es directamente la variable de interés, que se calibra con la equivalente local observada. En el contexto de cambio climático, se hace típicamente calibrando correcciones que ajustan parámetros de la distribución (por ejemplo, la media o percentiles), que generalmente se conocen como corrección de sesgo (en base a la distribución) (BC). Es importante señalar que la elección de reanálisis constituye una fuente adicional de incertidumbre para los métodos de PP, aunque no desempeña un papel importante en la península ibérica (Brands et al., 2012).

Gutiérrez et al. (2013) y San-Martín et al. (2017) evaluaron varios métodos convencionales de PP en España, incluyendo diferentes implementaciones del método de análogos, clasificación en tipos de tiempo y técnicas de regresión. Tras un proceso de selección analizando diferentes dominios y considerando distintas combinaciones de los predictores comúnmente utilizados, se comprobó que los resultados son más sensibles a la elección del predictor que al dominio geográfico, aunque generalmente se obtienen mejores resultados con dominios más pequeños cubriendo la península ibérica. En este caso se sugiere que la mejor configuración de predictores está formada por la presión a nivel del mar y la temperatura a 2 metros para pronosticar la temperatura, y la presión a nivel del mar, temperatura a 2 metros y la humedad específica a $850 \mathrm{hPa}$ para la precipitación. Se demostró que los MCG del CMIP5 son capaces de simular bien estos predictores a gran escala (Brands et al., 2013). Además, se han examinado otros métodos de regionalización estadística de PP en la península ibérica, incluyendo un método de análogos de dos pasos (Ribalaygua et al., 2013) y una técnica estocástica de tipos de tiempo para precipitación (Osca et al., 2013). Estos estudios se llevaron a cabo en el marco del proyecto ESTCENA, dentro del plan nacional español de escenarios regionalizados. Casanueva et al. (2016b) analizaron los sesgos de los MCR de EURO-CORDEX en la península ibérica y el efecto de los métodos de $\mathrm{BC}$ basados en correcciones sencillas (relacionadas con la intensidad y frecuencia de la precipitación). Además, se han analizado técnicas más sofisticadas de corrección de sesgo en la península ibérica (Amengual et al., 2011), así como en el contexto de estudios a escala continental (Dosio y Paruolo, 2011).

Son interesantes los trabajos que combinan ambas técnicas (estadísticas y dinámicas). Turco et al. (2011) y Turco et al. (2017) analizaron el potencial de la regionalización estadística para la corrección de sesgo mediante los métodos MOS genéricos (basados en análogos), a partir de la correspondencia marginal diaria presentada por las simulaciones MCR alimentadas con reanálisis. Se mostró que estos métodos son una alternativa espacialmente coherente frente a los métodos de corrección de sesgos estándar, aunque la incapacidad de extrapolar para generar nuevos valores extremos implica que estos resultados se deban tomar con precaución en aplicaciones en las que este aspecto es relevante. Entre los beneficios de los MRE, cabe señalar que son menos costosos que los MCR desde el punto de vista computacional y permiten el pronóstico de variables no meteorológicas, como la energía eólica (García-Bustamante et al., 2013) o índices climáticos de impacto (Casanueva et al., 2014). La principal limitación de los MRE es que dependen de la serie temporal observada disponible para la fase de entrenamiento/calibración. Además, asumen que las relaciones estadísticas son invariables en el tiempo (hipótesis de estacionariedad) y pueden sufrir de la falta de capacidad de extrapolación cuando se utilizan en aplicaciones de cambio climático. Un ejemplo de esta limitación se puede encontrar en Gutiérrez et al. (2013) para métodos basados 
en análogos sobre la península ibérica. Finalmente, es importante destacar que se han llevado a cabo varios estudios que comparan los métodos de regionalización estadísticos y dinámicos en la península ibérica (Casanueva et al., 2013, San-Martín et al., 2017). Es necesario hacer notar que para una comparación justa, se debe tener especial cuidado en utilizar índices adecuados que no estén calibrados directamente por ninguna de las dos metodologías (Casanueva et al., 2016a).

\section{Referencias}

Amengual, A., Homar, V., Romero, R., Alonso, S., Ramis, C., 2011: A Statistical Adjustment of Regional Climate Model Outputs to Local Scales: Application to Platja de Palma, Spain. J. Climate, 25 (3), 939-957.

Argüeso, D., Hidalgo-Muñoz, J. M., Gámiz-Fortis, S. R., Esteban-Parra, M. J., Castro-Díez, Y., 2012: Evaluation of WRF mean y extreme precipitation over spain: present climate (1970-99). J. Climate, 25 (14), 4883-4897.

Argüeso, D., Hidalgo-Muñoz, J. M., Gámiz-Fortis, S. R., Esteban-Parra, M. J., Dudhia, J., Castro-Díez, Y., 2011: Evaluation of WRF parameterizations for climate studies over southern spain using a multistep regionalization. J. Climate, 24 (21), 5633-5651.

Baró, R., Lorente-Plazas, R., Montávez, J., JiménezGuerrero, P., 2017: Biomass burning aerosol impact on surface winds during the 2010 russian heat wave. Geophys. Res. Lett., 44 (2), 1088-1094.

Brands, S., Gutiérrez, J. M., Herrera, S., Cofiño, A. S., 2012: On the Use of Reanalysis Data for Downscaling. J. Climate, 25 (7), 2517-2526.

Brands, S., Herrera, S., Fernández, J., Gutiérrez, J. M., 2013: How well do CMIP5 Earth System Models simulate present climate conditions in Europe and Africa? Clim. Dyn., 41, 803-817.

Casanueva, A., Frías, M. D., Herrera, S., San-Martín, D., Zaninovic, K., Gutiérrez, J. M., 2014: Statistical downscaling of climate impact indices: testing the direct approach. Climatic Change, 127 (3-4), 547-560.

Casanueva, A., Herrera, S., Fernández, J., Frías, M. D., Gutiérrez, J. M., 2013: Evaluation and projection of daily temperature percentiles from statistical and dynamical downscaling methods. Nat. Haz. Earth Syst. Sci., 13 (8), 2089-2099.

Casanueva, A., Herrera, S., Fernández, J., Gutiérrez, J. M., 2016a: Towards a fair comparison of statistical and dynamical downscaling in the framework of the EUROCORDEX initiative. Climatic Change, 137, 411-426.

Casanueva, A., Kotlarski, S., Herrera, S., Fernández, J., Gutiérrez, J., Boberg, F., Colette, A., Christensen, O., Goergen, K., Jacob, D., Keuler, K., Nikulin, G., Teichmann, C., Vautard, R., 2016b: Daily precipitation statistics in a
EURO-CORDEX RCM ensemble: added value of raw and bias-corrected high-resolution simulations. Clim. Dyn., 47 (3-4), 719-737.

Casanueva, A., Bedia, J., Herrera, S., Fernández, J., Gutiérrez, J. M., 2018: Bias correction of multi-variate climate indices: A new diagnostic tool to assess the effect on the climate change signal, Climatic Change, 10.1007/ s10584-018-2167-5.

Déqué, M., Somot, S., Sánchez-Gómez, E., Goodess, C. M., Jacob, D., Lenderink, G., Christensen, O. B., 2012: The spread amongst ENSEMBLES regional scenarios: regional climate models, driving general circulation models and interannual variability. Clim. Dyn., 38 (5-6), 951-964.

Domínguez, M., Romera, R., Sánchez, E., Fita, L., Fernández, J., Jiménez-Guerrero, P., Montávez, J. P., Cabos, W. D., Liguori, G., Gaertner, M. Á., 2013: Present-climate precipitation and temperature extremes over spain from a set of high resolution RCMs. Clim. Res., 58 (2), 149-164.

Dosio, A. y Paruolo, P., 2011: Bias correction of the ENSEMBLES high-resolution climate change projections for use by impact models: Evaluation on the present climate. J. Geophys. Res.: Atmospheres, 116 (D16).

Fernández, J., Montávez, J., Sáenz, J., González-Rouco, J. F., Zorita, E., 2007: Sensitivity of the MM5 mesoscale model to physical parameterizations for regional climate studies: Annual cycle. J. Geophys. Res.: Atmospheres, 112 (D4).

Gaertner, M. Á., González-Alemán, J. J., Romera, R., Domínguez, M., Gil, V., Sánchez, E., Gallardo, C., Miglietta, M. M., Walsh, K. J., Sein, D. V. et al., 2016: Simulation of medicanes over the mediterranean sea in a regional climate model ensemble: impact of ocean-atmosphere coupling and increased resolution. Clim. Dyn., pp. 1-17.

García-Bustamante, E., González-Rouco, J. F., Navarro, J., Xoplaki, E., Luterbacher, J., Jiménez, P. A., Montávez, J. P., Hidalgo, A., Lucio-Eceiza, E. E., 2013: Relationship between wind power production and North Atlantic atmospheric circulation over the northeastern Iberian Peninsula. Clim. Dyn., 40 (3-4), 935-949.

García-Díez, M., Fernández, J., Fita, L., Yagüe, C., 2013: Seasonal dependence of WRF model biases and sensitivity to PBL schemes over europe. Q. J. R. Met. Soc., 139 (671), 501-514.

García-Díez, M., Fernández, J., San-Martín, D., Herrera, S., Gutiérrez, J., 2015a: Assessing and improving the local added value of wrf for wind downscaling. J. Appl. Meteor. Climatol., 54 (7), 1556-1568.

García-Díez, M., Fernández, J., Vautard, R., 2015b: A RCM multi-physics ensemble over europe: multi-variable evaluation to avoid error compensation. Clim. Dyn., 45 (11-12), 3141-3156. 
Gómez, G., Cabos, W. D., Liguori, G., Sein, D., LozanoGaleana, S., Fita, L., Fernández, J., Magariño, M. E., JiménezGuerrero, P., Montávez, J. P., Domínguez, M., Romera, R., Gaertner, M. Á., 2016: Characterization of the wind speed variability and future change in the Iberian Peninsula and the balearic islands. Wind Energy, 19 (7), 1223-1237.

Gómez-Navarro, J., Montávez, J., Jerez, S., Jiménez-Guerrero, P., Lorente-Plazas, R., González-Rouco, J. F., Zorita, E., 2011: A regional climate simulation over the Iberian Peninsula for the last millennium. Clim. Past., 7, 451-472.

Gómez-Navarro, J., Montávez, J., Jerez, S., JiménezGuerrero, P., Zorita, E., 2012: What is the role of the observational dataset in the evaluation and scoring of climate models? Geophys. Res. Lett., 39 (24).

Gómez-Navarro, J., Montávez, J., Wagner, S., Zorita, E., 2013: A regional climate palaeosimulation for europe in the period 1500-1990, part 1: Model validation. Clim. Past, $9(4), 1667-1682$.

Gutiérrez, J. M., San-Martín, D., Brands, S., Manzanas, R., Herrera, S., 2013: Reassessing Statistical Downscaling Techniques for Their Robust Application under Climate Change Conditions. J. Climate, 26 (1), 171-188.

Herrera, S., Fernández, J., Gutiérrez, J. M., 2016: Update of the Spain02 gridded observational dataset for EUROCORDEX evaluation: assessing the effect of the interpolation methodology. Int. J. Climatol., 36 (2), 900-908.

Herrera, S., Fita, L., Fernández, J., Gutiérrez, J. M., 2010: Evaluation of the mean and extreme precipitation regimes from the ensembles regional climate multimodel simulations over Spain. J. Geoph. Res.: Atmosph., 115 (D21).

Jacob, D., Petersen, J., Eggert, B., Alias, A., Christensen, O. B., Bouwer, L. M., Braun, A., Colette, A., Déqué, M., Georgievski, G., Georgopoulou, E., Gobiet, A., Menut, L., Nikulin, G., Haensler, A., Hempelmann, N., Jones, C., Keuler, K., Kovats, S., Krner, N., Kotlarski, S., Kriegsmann, A., Martin, E., Meijgaard, E. von, Moseley, C., Pfeifer, S., Preuschmann, S., Radermacher, C., Radtke, K., Rechid, D., Rounsevell, M., Samuelsson, P., Somot, S., Soussana, J.F., Teichmann, C., Valentini, R., Vautard,, R., Weber, B., Yiou, P., 2014: EURO-CORDEX: new high-resolution climate change projections for European impact research. Regional Environmental Change, 14 (2), 563-578.

Jerez, S., Montávez, J., Gómez-Navarro, J., Jiménez, P., Jiménez-Guerrero, P., Lorente, R., y González-Rouco, J. F., 2012: The role of the land-surface model for climate change projections over the iberian peninsula. J. Geophys. Res.: Atmospheres, 117 (D1).

Jerez, S., Montávez, J. P., Gómez-Navarro, J. J., Jiménez-Guerrero, P., Jiménez, J., González-Rouco, J. F., 2010: Temperature sensitivity to the land-surface model in MM5 climate simulations over the iberian peninsula. Meteorologische zeitschrift, 19 (4), 363-374.
Jerez, S., Montávez, J. P., Gómez-Navarro, J. J., LorentePlazas, R., García-Valero, J. A., Jiménez-Guerrero, P., 2013a: A multi-physics ensemble of regional climate change projections over the Iberian Peninsula. Clim. Dyn., 41 (7-8), 1749-1768.

Jerez, S., Montávez, J. P., Jiménez-Guerrero, P., GómezNavarro, J. J., Lorente-Plazas, L., Zorita, E., 2013b: A multiphysics ensemble of present-day climate regional simulations over the iberian peninsula. Clim. Dyn., 40 (11-12), 3023-3046.

Jerez, S., Trigo, R. M., Vicente-Serrano, S. M., Pozo-Vázquez, D., Lorente-Plazas, R., Lorenzo-Lacruz, J., Santos-Alamillos, F., Montávez, J., 2013c: The impact of the north atlantic oscillation on renewable energy resources in southwestern Europe. J. Appl. Meteor. Climatol., 52 (10), 2204-2225.

Jiménez, P. A., González-Rouco, J. F., García-Bustamante, E., Navarro, J., Montávez, J. P., de Arellano, J. V.-G., Dudhia, J., y Muñoz-Roldán, A., 2010: Surface wind regionalization over complex terrain: Evaluation and analysis of a high-resolution WRF simulation. J. Appl. Meteor. Climatol., 49 (2), 268-287.

Jiménez-Guerrero, P., Montávez, J., Domínguez, M., Romera, R., Fita, L., Fernández, J., Cabos, W., Liguori, G., Gaertner, M., 2013: Mean fields and interannual variability in RCM simulations over Spain: the ESCENA project. Clim. Res., 57 (3), 201-220.

Katragkou, E., García-Díez, M., Vautard, R., Sobolowski, S., Zanis, P., Alexandri, G., Cardoso, R., Colette, A., Fernández, J., Gobiet, A. et al., 2015: Regional climate hindcast simulations within EURO-CORDEX: evaluation of a WRF multi-physics ensemble. Geosc. Model develop., 8 (3), 603-618.

Knist, S., Goergen, K., Buonomo, E., Christensen, O. B., Colette, A., Cardoso, R. M., Fealy, R., Fernández, J., GarcíaDíez, M., Jacob, D. et al., 2017: Land-atmosphere coupling in EURO-CORDEX evaluation experiments. J. Geophys. Res.: Atmospheres. doi: 10.1002/2016JD025476.

Kotlarski, S., Keuler, K., Christensen, O. B., Colette, A., Déqué, M., Gobiet, A., Goergen, K., Jacob, D., Lüthi, D., Meijgaard, E. van, Nikulin, G., Schär, C., Teichmann, C., Vautard, R., Warrach-Sagi, K., Wulfmeyer, V., 2014: Regional climate modeling on european scales: a joint standard evaluation of the EURO-CORDEX RCM ensemble. Geosc. Model Develop., 7 (4), 1297-1333.

López-Franca, N., Sánchez, E., Domínguez, M., 2013: Changes in the onset and length of seasons from an ensemble of regional climate models over Spain for future climate conditions. Theor. Appl. Climatol., 114 (3-4), 635-642.

López-Franca, N., Sánchez, E., Losada, T., Domínguez, M., Romera, R., Gaertner, M. Á., 2015: Markovian characteristics of dry spells over the iberian peninsula under present and future conditions using escena ensemble of regional climate models. Clim. Dyn., 45 (3-4), 661-677. 
Lorente-Plazas, R., Montávez, J., Jerez, S., GómezNavarro, J., Jiménez-Guerrero, P., Jiménez, P., 2015: A 49 year hindcast of surface winds over the iberian peninsula. Int. J. Climatol., 35 (10), 3007-3023.

Maraun, D., Widmann, M., Gutiérrez, J. M., Kotlarski, S., Chandler, R. E., Hertig, E., Wibig, J., Huth, R., Wilcke, R. A., 2015: VALUE: A framework to validate downscaling approaches for climate change studies. Earth's Future, 3 (1), 2014EF000259.

Osca, J., Romero, R., Alonso, S., 2013: Precipitation projections for Spain by means of a weather typing statistical method. Global Planet. Change, 109, 46-63.

Palacios-Peña, L., Baró, R., Guerrero-Rascado, J. L., Alados-Arboledas, L., Brunner, D., Jiménez-Guerrero, P., 2017: Evaluating the representation of aerosol optical properties using an online coupled model over the iberian peninsula. Atmospheric Chemistry and Physics, 17 (1), 277.

Pons, M. R., Herrera, S., Gutiérrez, J. M., 2016: Future trends of snowfall days in northern Spain from ENSEMBLES regional climate projections. Clim. Dyn., 46 (11-12), 3645-3655.

Prein, A., Gobiet, A., Truhetz, H., Keuler, K., Goergen, K., Teichmann, C., Maule, C. F., Meijgaard, E. van, Déqué, M., Nikulin, G. et al., 2016: Precipitation in the EUROCORDEX $0.11^{\circ}$ and $0.44^{\circ}$ simulations: high resolution, high benefits? Clim. Dyn., 46 (1-2), 383-412.

Ribalaygua, J., Torres, L., Pórtoles, J., Monjo, R., Gaitán, E., Pino, M. R., 2013: Description and validation of a twostep analogue/regression downscaling method. Theor. Appl. Climatol., 114 (1-2), 253-269.

Ruiz-Arias, J. A., Dudhia, J., Santos-Alamillos, F. J., PozoVázquez, D., 2013: Surface clear-sky shortwave radiative closure intercomparisons in the weather research and forecasting model. J. Geophys. Res.: Atmospheres, 118 (17), 9901-9913.

San-Martín, D., Manzanas, R., Brands, S., Herrera, S., Gutiérrez, J. M., 2017: Reassessing Model Uncertainty for Regional Projections of Precipitation with an Ensemble of Statistical Downscaling Methods. J. Climate, 30 (1), 203-223.

Sánchez, E. y Míguez-Macho, G., 2010: Regional climate projections over the Iberian Peninsula: climate change scenarios modeling. CLIVAR-Spain Regional climate change assessment report: Climate in Spain: Past, Present and $\mathrm{Fu}-$ ture (Editors: F. Pérez, Fiz y Boscolo, Roberta) pp. 69-80. Disponible en http://www.clivar.org/documents/spain-2010

Sánchez, E., Domínguez, M., Romera, R., López-Franca, N., Gaertner, M. Á., Gallardo, C., Castro, M., 2011: Regional modeling of dry spells over the iberian peninsula for present climate and climate change conditions. Climatic change, 107 (3), 625-634.

Sotillo, M., Ratsimandresy, A., Carretero, J., Bentamy, A., Valero, F., González-Rouco, F., 2005: A high-resolution 44-year atmospheric hindcast for the mediterranean basin: contribution to the regional improvement of global reanalysis. Clim. Dyn., 25 (2-3), 219-236.

Turco, M., Llasat, M. C., Herrera, S., Gutiérrez, J. M., 2017: Bias correction and downscaling of future RCM precipitation projections using a MOS? Analog technique. J. Geophys. Res.: Atmospheres, 122 (5), 2631-2648.

Turco, M., Quintana-Seguí, P., Llasat, M. C., Herrera, S., Gutiérrez, J. M., 2011: Testing MOS precipitation downscaling for ENSEMBLES regional climate models over Spain. J. Geophys. Res., 116 (D18).

Turco, M., Sanna, A., Herrera, S., Llasat, M.-C., Gutiérrez, J. M., 2013: Large biases and inconsistent climate change signals in ensembles regional projections. Climatic Change, $120(4), 859-869$.

Vautard, R., Gobiet, A., Jacob, D., Belda, M., Colette, A., Déqué, M., Fernández, J., García-Diez, M., Goergen, K., Guttler, I. et al., 2013: The simulation of european heat waves from an ensemble of regional climate models within the EURO-CORDEX project. Clim. Dyn., 41 (9-10), 2555-2575. 